\title{
Trade-Offs in Cryptographic Implementations of Temporal Access Control
}

\author{
Jason Crampton \\ Information Security Group, Royal Holloway, University of London \\ jason.crampton@rhul.ac.uk
}

\begin{abstract}
In recent years, we have seen the development of key assignment schemes that use cryptography to enforce time-based authorization policies. One of the most important aspects of these schemes is the balance between the time required to derive keys and the amount of storage required for the public information from which keys are derived. The derivation time and storage are dependent on the number of time periods used in the authorization policy. In this paper, we discuss novel schemes that achieve good trade-offs between these competing parameters and for which explicit bounds can be given in terms of the number of time periods.
\end{abstract}

\section{Motivation}

In some situations, we may wish to use cryptographic techniques to implement some form of access control. By (symmetrically) encrypting the contents of a data object, we restrict access to an object to those users who possess the corresponding encryption key. The problem we now need to solve is the efficient and accurate distribution of encryption keys to authorized users.

In recent years, there has been a considerable amount of interest in key encrypting schemes. In such schemes, a user is given a secret value - typically a single key - which enables the user to derive some collection of encryption keys. Key derivation is performed using the secret value and some information made publicly available by the scheme administrator.

The most widely studied situation assumes the existence of a partially ordered set $(L, \leqslant)$ and an encryption key $\kappa(x)$ associated with each element $x \in L$. Each user is associated with a security label $\lambda(u) \in L$ and should be able to derive all keys in the set $\{\kappa(y): y \leqslant \lambda(u)\}$. This scenario is based on the information flow policies for confidentiality that were widely studied in the 1970s [5,12]. It is generally assumed that the trivial solution in which the user's secret is the set of keys $\{\kappa(y): y \leqslant \lambda(u)\}$ is not of interest. Instead, the research literature has focused on so-called key assignment schemes in which each user is given a single key and additional public information is used to derive additional keys. The two objectives when designing such a scheme are to minimize the amount of public information and the time required to derive a key. Unsurprisingly, it is not possible to realize both objectives simultaneously, so trade-offs have been 
sought. Crampton et al. provide a survey of, and taxonomy for, key assignment schemes, and the various factors that affect the parameters described above [9].

At the same time, we have seen the development of access control models in which time plays an important role in deciding whether access requests are authorized or not [6]. One particular application of such "temporal access control" systems is the protection of data that is made available periodically as (part of) a subscription-based service [7].

Crampton et al. suggested that temporal access control policies, in which there are number of time points and users were authorized to access data over a sequence of consecutive time points, could be implemented using key assignment schemes. Shortly after, Atallah et al. [2], Ateniese et al. [4] and De Santis et al. [11] described key assignment schemes for temporal access control using this representation for time. Their work focused on two particular aspects:

- the development of schemes that were secure against key recovery, and

- the reduction of the storage required for public information and the number of operations required for key derivation.

One shortcoming of their work is that the approaches used to tackle the second of these issues do not consider the actual requirements of the underlying access control model. Instead, generic techniques to reduce the diameter of a directed graph are applied. This has two consequences: application-specific optimizations are not considered and only the asymptotic behavior of the constructions is provided. Given that the number of time intervals is likely to be rather small in many practical applications, it is not clear that this kind of approach is the most appropriate.

In this paper, we consider application-specific optimizations that arise from two rather straightforward observations. This enables us to present concrete schemes with precise bounds on the amount of storage and the number of derivation steps required. One nice feature of this work is that we may still be able to exploit the techniques used in earlier work to obtain some further reduction in storage and derivation time.

In the next section, we describe some relevant background material. We then make a simple, yet powerful, observation and explore the schemes that can be derived as result of this observation. In Sect. 4, we explore the consequences of another simplification. Section 5 describes a different way of decomposing the partially ordered set of time intervals, which yields methods for fixing the number of derivation steps required. We conclude the paper with a summary of our contributions, a comparison with related work and some suggestions for future work.

\section{Key Assignment Schemes}

Let us suppose we have a partially ordered set of security labels $(L, \leqslant)$. An information flow policy requires that each user $u$ and protected object $o$ be assigned a security label; $u$ is authorized to read $o$ provided the security label 
of $u$ is greater than or equal to that of $o$. More formally, let $\lambda: U \cup O \rightarrow L$ be a labeling function that associates each entity with a security label. Then $u$ is authorized to access $o$ if and only if $\lambda(u) \geqslant \lambda(o)$.

A key assignment scheme may be used to enforce an information flow policy. A key assignment scheme comprises a set of keys $\{\kappa(x): x \in L\}$ and a set of public information. Each object with security label $x$ is encrypted with $\kappa(x)$. A user $u$ with the key $\kappa(\lambda(u))$ must be able to derive $k(y)$ for any $y \leqslant \lambda(u)$ using $\kappa(\lambda(u))$ and public information. Hence, a user can decrypt any object with security label $\lambda(y)$, where $y \leqslant \lambda(u)$. The parameters that characterize the behavior of a key assignment scheme are [9]:

- the number of keys that a user requires;

- the amount of public information that is required;

- the amount of time taken to derive a key.

Generally speaking, we are only interested in schemes in which each user has a single key. (We could, trivially, give a user a key for each of the labels that are less than or equal to $\lambda(u)$ - that is, the set of keys $\{\kappa(y): y \leqslant \lambda(u)\}-$ but this type of approach is rarely considered appropriate.) As a consequence we require either more public information or longer key derivation times (or both).

\subsection{Key Assignment Schemes for Directed Graphs}

A partially ordered set $(L, \leqslant)$ can be represented by a graph $(L, E)$. There are two obvious choices for the edge set $E$ : one is the full partial order relation $\leqslant$; the second option is to omit all transitive and reflexive edges from $\leqslant$ to obtain the covering relation, denoted $\lessdot$. The graph $(L, \lessdot)$ is called the Hasse diagram of $L$, and is the usual representation of $L$ as a directed graph [10].

Note that we can easily generate a key assignment scheme for any directed acyclic graph $(V, E)$. Specifically, we associate a key $\kappa(x)$ with each $x \in V$, and, for each edge $(x, y) \in E$, we publish $E n c_{\kappa(x)}(\kappa(y))$, where $E n c_{k}(m)$ denotes the encryption of message $m$ using key $k{ }^{1}$ Then any user in possession of $\kappa(x)$ can derive $\kappa(y)$ in one step, and for any $z$ on a path from $x$ containing $m$ edges, $\kappa(z)$ can be (iteratively) derived in $m$ steps.

\subsection{Trade-Offs in Key Assignment Schemes}

It can seen that key assignments schemes for directed graphs can be used specifically for information flow policies. We may use the graph $(L, \leqslant)$, in which case key derivation can always be performed in one step. In contrast, key derivation may require a number of steps if we use the graph $(L, \lessdot)$. The trade-off here is that the second graph contains fewer edges and hence the number of items of public information that are required to support key derivation is smaller. The study of these kinds of trade-offs will be the focus of this paper.

\footnotetext{
${ }^{1}$ Atallah et al., for example, use the "encryption" $\kappa(y) \oplus h(\kappa(x) \| y)$, where $h$ is a hash function, $\oplus$ is bit-wise XOR, and $\|$ denotes concatenation of strings [3].
} 


\subsection{Security Considerations}

A number of schemes exist in the literature that prevent a user from obtaining keys for which she is not authorized by colluding with other users. The first such scheme was developed by Akl and Taylor [1] specifically for partially ordered sets. Since then Atallah et al. [2] and, independently, Ateniese et al. [4] have shown that there exist key assignment schemes that are secure against "key recovery" and can be used with arbitrary directed graphs. We will assume henceforth that the schemes we propose, which only vary in the choice of edge set for the graph, will be implemented using a scheme that is secure against key recovery.

\subsection{Key Assignment Schemes for Temporal Access Control}

Let us now assume that users are authorized to access objects for specified periods of time. We assume that there exists a sequence of time "points" $t_{1}, \ldots, t_{m}$ and that there are cryptographic keys $\kappa_{1}, \ldots, \kappa_{m}$ associated with each time point. Each key $\kappa_{i}$ is used to encrypt data (using some appropriate symmetric encryption technique) released at time point $i$. Each time point is a collection of time instants; that is, a time interval. A sequence of consecutive time points represents a longer time interval.

We can use a key assignment scheme to enforce a temporal access control scheme. Specifically, the lattice of security labels is the set of intervals defined over the set $\left\{t_{1}, \ldots, t_{m}\right\}$ ordered by subset inclusion, which we denote by $I_{m}$. That is, $I_{m}=\left\{\left[t_{i}, t_{j}\right]: 1 \leqslant i \leqslant j \leqslant m\right\}$, where $\left[t_{i}, t_{j}\right]$ denotes the set $\left\{t_{i}, t_{i+1}, \ldots, t_{j-1}, t_{j}\right\}$. Clearly, we may represent $\left\{t_{1}, \ldots, t_{m}\right\}$ simply as the set $\{1, \ldots, m\}$, which we denote by $[m]$. Henceforth, we use this representation of, and notation for, the set of time points: a user authorized for time points $i, \ldots, j$ is assigned to label $[i, j]$; key (object) $\kappa_{i}$ is assigned to label $[i, i]$.

Note that the number of edges in the graph $\left(I_{m}, \lessdot\right)$ contains $m(m-1)$ edges. Note also that the Hasse diagram of $I_{m}$ can be represented as a triangular grid, with the apex of the triangle representing the interval $[1, m]$ and the long diagonal representing the intervals $[i, i] .^{2}$ This graphical interpretation of $I_{4}$ is illustrated in Fig. 3(a) on page 7. Using this graph, we can see that iterative key derivation requires precisely $m-1$ steps to get from vertex $[1, m]$ to any vertex of the form $[i, i]$. More generally, it requires $j-i$ steps to get from the vertex $[i, j]$ to a vertex $[l, l]$, where $l \in[i, j]$.

Henceforth, we will call the triangular pattern of side $m$ an $m$-triangle, denoted by $T_{m}$. We will write $D_{m}$ to denote an $m$-diamond - a diamond-shaped pattern of side $m$ (obtained by joining two copies of $T_{m}$ along the long diagonal). $T_{4}$ and $D_{4}$ are illustrated in Fig. 1 . Nodes of the form $[i, i] \in T_{m}$ will be called leaf nodes.

To conclude this section, we summarize the problem we address in the remainder of the paper. We wish to construct a graph $\left(T_{m}, E_{m}\right)$, such that

\footnotetext{
${ }^{2}$ All edges in all graphs depicted in this paper are assumed to point down the page.
} 


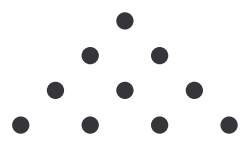

(a) $T_{4}$

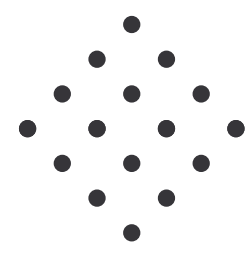

(b) $D_{4}$

Fig. 1. $T_{4}$ and $D_{4}$

- for any non-leaf node $[i, j]$ there exists a path from $[i, j]$ to $[k, k]$ for every $k \in[i, j]$

- $\left|E_{m}\right|$ is small;

- the length of the longest path in $\left(T_{m}, E_{m}\right)$ is small.

The first criterion simply reflects that the graph must implement the desired access control policy. The second means that we wish to keep the public storage requirements small, while the final criterion means that we wish to make the worst-case key derivation time small. Note that $T_{m}$ is fixed, so any scheme is defined by the choice of $E_{m}$. For brevity, we will simply refer to a scheme $E_{m}$ (with $T_{m}$ and the graph $\left(T_{m}, E_{m}\right)$ left implicit).

\section{An Immediate Improvement}

We start by making the first of our application-specific observations. This immediately yields a number of significant improvements to the number of steps required.

Remark 1 The only security labels for which a user needs to derive a key have the form $[i, i]$ (since no objects are associated with any interval of the form $[i, j]$, where $i<j)$. Therefore, it is irrelevant whether a user with security label $[i, j]$ can derive $\left[i^{\prime}, j^{\prime}\right]$, where $\left[i^{\prime}, j^{\prime}\right] \subseteq[i, j]$ and $i^{\prime} \neq j^{\prime}$.

This means that we can seek to optimize the key assignment scheme used to derive keys by omitting some of the edges in the triangular grid $I_{m}$. Clearly, the triangular grid is "self-similar", so it is natural to examine recursive methods for generating graphs in which the number of hops is reduced. Before we discuss such methods, we introduce a very simple 1-hop scheme.

Scheme 1 (1-hop derivation) In our first scheme $E_{m}^{(1)}$, we simply insert an edge between every non-leaf node $[i, j]$ to leaf node $[k, k]$, for all $k \in[i, j]$.

Scheme $E_{4}^{(1)}$ is illustrated in Fig. 2. We can see that 16 edges are required: 4 for the top node, 3 for each of the nodes in the second row, and 2 for each of the nodes in the third row. 


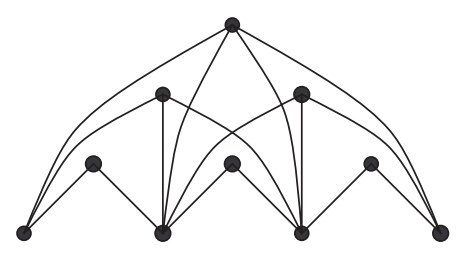

Fig. 2. The scheme $E_{4}^{(1)}$

Proposition 2 For all $m \geqslant 1, e_{m}^{(1)}=\frac{1}{6} m(m-1)(m+4)$, where $e_{m}^{(1)}$ denotes the cardinality of $E_{m}^{(1)}$.

Proof. It is easy to see that we require $m$ edges for the uppermost node, $m-1$ edges for each of the two nodes in the next row, etc. Hence, we see that the number of edges required is given by

$$
\begin{aligned}
\sum_{i=1}^{n-1} i(n-i+1) & =(n+1) \sum_{i=1}^{n-1} i-\sum_{i=1}^{n-1} i^{2} \\
& =\frac{1}{2}(n+1)(n-1) n-\frac{1}{6} n(n-1)(2 n-1) \\
& =\frac{1}{6} n(n-1)(3 n+3-(2 n-1)) \\
& =\frac{1}{6} n(n-1)(n+4)
\end{aligned}
$$

Scheme 2 ( $\log _{2} m$-hop derivation) To construct our second scheme $E_{m}^{(2)}$, we make the following observations:

- $T_{2 m}$ contains two copies of $T_{m}$ and one copy of $D_{m}$;

- no edges are required between nodes in $D_{m}$ (by Remark 1);

- $I_{m}$ contains two edges for each node in $D_{m}$

- for each node $[i, j] \in D_{m}, m, m+1 \in[i, j]$.

Hence, to construct $E_{m}^{(2)}$, we replace the two edges in $I_{m}$ from each node $[i, j] \in$ $D_{m}$ with two edges $([i, j],[i, m])$ and $([i, j],[m+1, j])$. In other words, each node in $D_{m}$ is directly connected to a node in each copy of $T_{m}$.

Note that the number of derivation hops for $T_{2 m}$ is one more than that for $T_{m}$. Henceforth, we will write $h_{m}^{(i)}$ for the number of derivation hops in scheme $E_{m}^{(i)}$. In particular, we have $h_{2 m}^{(2)}=h_{m}^{(2)}+1$.

Figure 3(b) illustrates $E_{4}^{(2)}$. Note that it is not possible to get from the top node to a leaf node in 1 hop. Note, however, that only 12 edges are required. 


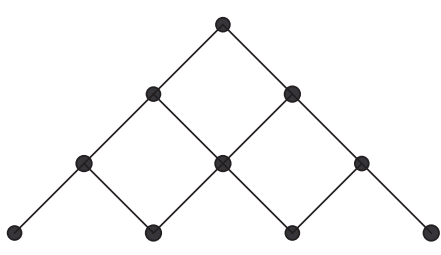

(a) $I_{4}$

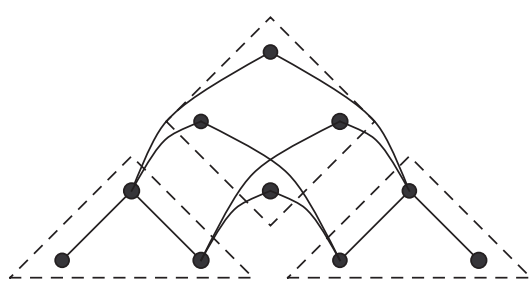

(b) $E_{4}^{(2)}$

Fig. 3. Constructing the scheme $E_{4}^{(2)}$

In an effort to keep the notation simple, we will write $h_{m}$ and $e_{m}$ (rather than $h_{m}^{(i)}$ and $e_{m}^{(i)}$ ) where no confusion can arise about which scheme is under consideration.

Proposition 3 For scheme $E_{n}^{(2)}, n \geqslant 2$, we have

$$
\begin{aligned}
e_{2 n} & =2 e_{n}+2 n^{2} \\
e_{n} & =n(n-1) \\
h_{n} & =\log _{2} n
\end{aligned}
$$

Proof. The first result follows immediately from the construction. Assuming we use scheme $E_{n}^{(2)}$, then we need two copies of $T_{n}$ each containing $e_{n}$ edges and we need two edges for each of the $n^{2}$ nodes in $D_{n}$.

To prove $(2)$, recall that $I_{n}$ contains $n(n-1)$ edges and note that the construction of $E_{n}^{(2)}$ replaces the two edges for each node in $D_{n}$ with two different edges. Hence, $e_{n}$ is the same as the number of edges in $I_{n}$.

Finally, note that $h_{2 n}=h_{n}+1$. Solving this recurrence relation, we obtain $h_{n}=\log _{2} n$.

The important thing to note about $E_{n}^{(2)}$ is that it requires no more storage than $I_{n}$, but the upper bound on key derivation is $\log _{2} n$ steps rather than $n-1$ steps. Finally, we note that our approach can also be applied to triangle $T_{m+n}$. We illustrate the scheme $E_{5}^{(2)}$ in Fig. 4.

\section{Skipping a Level}

Suppose we have a scheme for $T_{n}$ which requires no more than $h_{n}$ steps, and we have used Scheme 2 and the scheme for $T_{n}$ to construct a scheme for $T_{2 n}$ with $h_{n}+1$ steps. Now consider $T_{4 n}$, which breaks down into two copies of $T_{2 n}$ and one copy of $D_{2 n}$. Note that $D_{2 n}$ can be sub-divided into four copies of $D_{n}$. Nodes in each copy of $D_{n}$ can then be attached to nodes in the appropriate copies of 


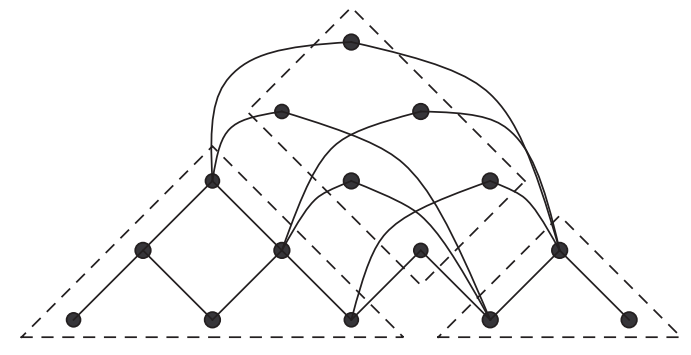

Fig. 4. Constructing $E_{5}^{(2)}$ using $T_{2}$ and $T_{3}$

$T_{n}$ contained within each $T_{2 n}$, skipping the intermediate nodes in $T_{2 n}$. Hence, we do not increase the total number of hops required for derivation: a node in $D_{2 n}$ is connected directly to a node in $T_{n}$, as are nodes in each copy of $T_{2 n}$. In other words, we have $h_{4 n}=h_{n}+1=h_{2 n}$. More formally, we have the following construction.

Scheme 3 Let $n=b m$. Then for $T_{2 n}$, we split $D_{n}$ into $b^{2}$ copies of $D_{m}$. We connect each node in each $D_{m}$ to the appropriate nodes in copies of $T_{m}$. In particular, for $[i, j]$, where $i=\alpha_{i} m+\beta_{i}$ and $j=\alpha_{j} m+\beta_{j}$, we add edges from $[i, j]$ to nodes $\left[i,\left(\alpha_{i}+1\right) m\right],\left[\left(\alpha_{i}+1\right) m+1,\left(\alpha_{i}+2\right) m\right], \ldots,\left[\alpha_{j} m, j\right]$.

The (partial) construction of $E_{8}^{(3)}$ is illustrated in Fig. 5. Node $[2,6]$ in $D_{4}$ has been connected to nodes $[2,2],[3,4]$ and $[5,6]$. Note that $[2,6]$ would have been connected to nodes $[2,4]$ and $[5,6]$ in $E_{8}^{(2)}$. Note also that $h_{8}^{(3)}=h_{4}^{(3)}=h_{2}^{(3)}+1$.

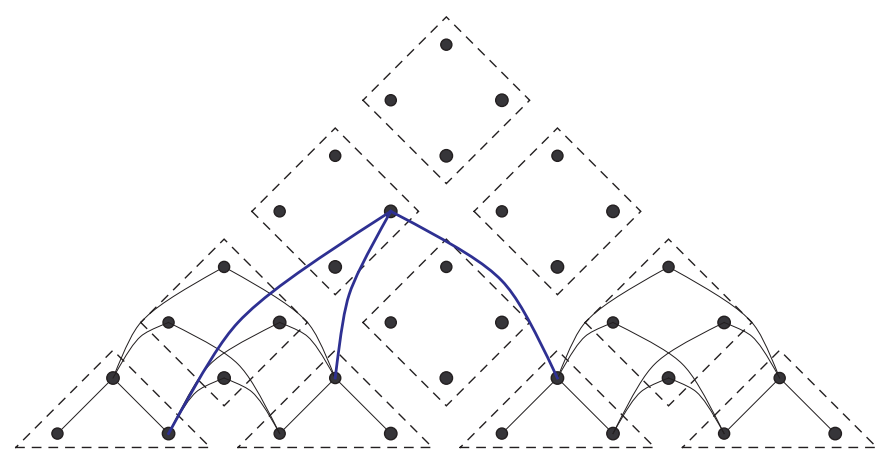

Fig. 5. The partial construction of $E_{8}^{(3)}$ 


\begin{tabular}{r|r|r|r}
$n$ & Steps & Derivation of $e_{n}$ & $e_{n}$ \\
\hline 2 & 1 & & 2 \\
4 & 1 & & 16 \\
8 & 2 & $2 e_{4}+\left(2^{0}+1\right) \cdot 4^{2}$ & 64 \\
16 & 2 & $2 e_{8}+\left(2^{1}+1\right) \cdot 8^{2}$ & 320 \\
32 & 3 & $2 e_{16}+\left(2^{0}+1\right) \cdot 16^{2}$ & 1152 \\
64 & 3 & $2 e_{32}+\left(2^{1}+1\right) \cdot 32^{2}$ & 5376 \\
128 & 4 & $2 e_{64}+\left(2^{0}+1\right) \cdot 64^{2}$ & 18944 \\
256 & 4 & $2 e_{128}+\left(2^{1}+1\right) \cdot 128^{2}$ & 87040
\end{tabular}

Table 1. Constructing a scheme with $\frac{1}{2} \log n$ derivation steps

Lemma 4 For scheme $E_{n}^{(3)}$, where $n=b m$, we have

$$
e_{2 n}=2 e_{n}+(b+1) n^{2} .
$$

Moreover, if $h_{n}>h_{m}$ then $h_{2 n}=h_{n}$.

Proof. Omitted due to space constraints.

Notice the trivial case $b=1$ corresponds to $e_{2 n}=2 e_{n}+2 n^{2}$. Of course, in this case we add a step to the derivation cost, because we do not skip a level.

We now apply this construction to the case $b=2$, in doing so deriving a scheme $E_{m}^{(4)}$ in which $h_{n}=\frac{1}{2} \log _{2} n$. We define schemes for $T_{2}$ and $T_{4}$, each of which use one derivation step. Then we can define a scheme for $T_{8}$ that requires two derivation steps using two copies of $T_{4}$ as the building blocks. We can define a scheme for $T_{16}$ that also requires two derivation steps using four copies of $T_{4}$ as the building blocks. Extending further, we define a scheme for $T_{32}$ that requires three derivation steps using two copies of $T_{16}$ as the building blocks, and define a scheme for $T_{64}$ that also requires three steps using four copies of $T_{16}$ as building blocks, etc. This construction is illustrated in Table 1 . Using the result of Lemma 4 and noting that $b=1$ or $b=2$ in this construction, we have $e_{2 n} \leqslant 2 e_{n}+3 n^{2}$.

Lemma 5 For $E_{n}^{(4)}$ and $n=2^{m}$, we have $e_{n} \leqslant \frac{3}{2} n(n-1)$.

Proof. We prove the result by induction on $m$. We choose a 1-hop scheme for $T_{4}$ with 16 edges. Hence, the result holds for $m=2$. Now let us assume that the result holds for $m \leqslant M$ and consider $e_{2^{M+1}}$. Then, by construction,

$$
\begin{aligned}
e_{2^{M+1}} & \leqslant 2 e_{2^{M}}+\frac{3}{2} 2^{2 M} \\
& \leqslant 2\left(\frac{3}{2}\left(2^{2 M}-2^{M}\right)\right)+\frac{3}{2} 2^{2 M} \quad \text { (by inductive hypothesis) } \\
& =\frac{3}{2}\left(3.2^{2 M}-2^{M+1}\right)<\frac{3}{2}\left(2^{2 M+2}-2^{M+1}\right)=\frac{3}{2} 2^{M+1}\left(2^{M+1}-1\right)
\end{aligned}
$$


as required.

We conjecture that we can use $b=2^{r}$, for any non-zero positive integer $r$, to derive a scheme with $h_{n} \approx \frac{1}{r+1} \log _{2} n$ and $e_{n} \leqslant \operatorname{an}(n-1)$, where $a$ is a function of $r$. The sole obstacle to the direct construction of a scheme analogous to $E_{n}^{(4)}$ is the construction of a scheme for the base case $T_{2^{r}}$ such that $e_{2^{r}} \leqslant \frac{1}{2} a 2^{r}\left(2^{r}-1\right)$. In particular, we will not be able to use a 1-hop scheme for $T_{2^{r}}$ (unlike the case $b=2$ ), because the number of edges in a 1-hop scheme for $T_{n}$ grows with the cube of $n$. However, it should always be possible to find a scheme for $T_{2^{r}}$ with a small number of hops so that the base case "works". This will be the subject of future work.

We also note that we can construct a scheme that only requires $\log _{2}\left(\log _{2} n\right)$ derivation steps. We define schemes for $T_{2}$ and $T_{4}$, each requiring a single derivation step. Then for $T_{8}=T_{2^{3}}$ and $T_{16}=T_{2^{4}}$ we build schemes using $T_{4}=T_{2^{2}}$. For $T_{2^{5}}, \ldots, T_{2^{8}}$, we build schemes using $T_{2^{4}}$. For $T_{2^{9}}, \ldots, T_{2^{16}}$, we build schemes using $T_{2^{8}}$. This construction is illustrated in Table 2. A similar technique can be applied for any $n=2^{2^{k}}$. In particular, we have the following result.

Lemma 6 Let $n=2^{2^{k}}$. Then we can construct a scheme for $T_{n}$ such that

$$
e_{n}-\sqrt{n} e_{\sqrt{n}}=\frac{1}{6} n \sqrt{n}(\sqrt{n}-1)(\sqrt{n}+4) \quad \text { and } \quad h_{n}=\log _{2}\left(\log _{2} n\right) .
$$

Proof. Omitted due to space constraints.

Using this formula, we have, for example,

$$
e_{256}=16 e_{16}+\frac{1}{6} \cdot 256 \cdot 16 \cdot 15 \cdot 20=209920,
$$

which can be confirmed by Table 2. Clearly, the number of edges required is approximately $\frac{1}{6} n^{2} \sqrt{n}$, since the term in $n^{2} \sqrt{n}$ will dominate the term in $\sqrt{n} e_{\sqrt{n}}$.

\section{A Multiplicative Decomposition of $T_{n}$}

Let $n=a b$. Then we can split $T_{n}$ into $b$ copies of $T_{a}$ and $t_{b-1}$ copies of $D_{a}$, where $t_{k}=\frac{1}{2} k(k+1)$ is the $k$ th triangle number. We treat the copies of $D_{a}$ and $T_{a}$ as "supernodes" in $T_{b}$. We can construct schemes for $T_{a}$ and $T_{b}$ and use $a^{2}$ copies of the scheme (one for each node in the non-leaf supernode) for $T_{b}$ to connect the set of $D_{a}$ s to the $T_{a}$ s. We then use the scheme for $T_{a}$ to derive keys within $T_{a}$. The construction is illustrated in Fig. 6 for $T_{12}$.

The simplest case arises when $b=2$ and gives rise to Scheme 2. In this case, we have two copies of $T_{a}$ and one copy of $D_{a}$. We use the obvious 1-hop scheme for $T_{2}$. We then recursively apply this construction to $T_{a}$. This process ultimately yields a scheme which requires no more than $\log _{2} n$ steps. 


\begin{tabular}{r|r|r|r|r}
$n$ & $\log _{2} n$ & $\left\lceil\log _{2}\left(\log _{2} n\right)\right.$ & Derivation of $e_{n}$ & $e_{n}$ \\
\hline 2 & 1 & 1 & & 2 \\
4 & 2 & 1 & & 16 \\
8 & 3 & 2 & $2 e_{4}+\left(2^{0}+1\right) \cdot 4^{2}$ & 64 \\
16 & 4 & 2 & $2 e_{8}+\left(2^{1}+1\right) .8^{2}$ & 320 \\
32 & 5 & 3 & $2 e_{16}+\left(2^{0}+1\right) \cdot 16^{2}$ & 1152 \\
64 & 6 & 3 & $2 e_{32}+\left(2^{1}+1\right) .32^{2}$ & 5376 \\
128 & 7 & 3 & $2 e_{64}+\left(2^{2}+1\right) \cdot 64^{2}$ & 31232 \\
256 & 8 & 3 & $2 e_{128}+\left(2^{3}+1\right) \cdot 128^{2}$ & 209920 \\
512 & 9 & 4 & $2 e_{256}+\left(2^{0}+1\right) \cdot 256^{2}$ & 550912 \\
1024 & 10 & 4 & $2 e_{512}+\left(2^{1}+1\right) .512^{2}$ & 1888256 \\
2048 & 11 & 4 & $2 e_{1024}+\left(2^{2}+1\right) \cdot 1024^{2}$ & 9019392 \\
4096 & 12 & 4 & $2 e_{2048}+\left(2^{3}+1\right) \cdot 2048^{2}$ & 55787520 \\
8192 & 13 & 4 & $2 e_{4096}+\left(2^{4}+1\right) .4096^{2}$ & 396787712 \\
16384 & 14 & 4 & $2 e_{8192}+\left(2^{5}+1\right) .8192^{2}$ & 3008167936 \\
32768 & 15 & 4 & $2 e_{16384}+\left(2^{6}+1\right) \cdot 16384^{2}$ & 23464640512 \\
65536 & 16 & 4 & $2 e_{32768}+\left(2^{7}+1\right) .32768^{2}$ & 185441976320
\end{tabular}

Table 2. Constructing a scheme with $\left\lceil\log _{2}\left(\log _{2} n\right)\right\rceil$ derivation steps

\subsection{A 2-Hop Scheme}

Let us now consider the case where we would like to construct a scheme that requires no more than 2 steps. Then we need 1-hop schemes for $T_{a}$ and $T_{b}$. Scheme 1 may be used to create such schemes requiring

$$
\frac{1}{6} a(a-1)(a+4) \text { and } \frac{1}{6} b(b-1)(b+4)
$$

edges, respectively. Hence, we can construct a scheme for $T_{a b}$ that requires no more than 2 steps, and the number of edges is

$a^{2} \frac{1}{6} b(b-1)(b+4)+b \frac{1}{6} a(a-1)(a+4)=\frac{1}{6} a b(a(b-1)(b+4)+(a-1)(a+4))$.

Returning to the construction of a 2-hop scheme for $T_{12}$ as illustrated in Fig. 6 , we divide $T_{12}$ into copies of $D_{4}$ and $T_{4}$, yielding a copy of $T_{3}$ in which the non-leaf supernodes are diamonds and leaf supernodes are triangles. We then construct a 1-hop scheme for $T_{3}$ using 6 edges (Fig. 6(b)). We duplicate this scheme for every node in the root supernode. Clearly, there are $4^{2}=16$ nodes in each supernode. Hence we require 6.16 such edges. Having done this, we can now get from any node that is contained in a copy of $D_{4}$ to a node in $T_{4}$ in one hop. It remains, therefore, to construct a scheme for each $T_{4}$ supernode such that we can get from any non-leaf node to a leaf node in one hop. Again, we can use Scheme 1 for this (as illustrated in Fig. 2). We require 16 edges for each of the three copies of $T_{4}$. The scheme therefore requires a total of $16.6+3.16=16.9=144$ edges. 


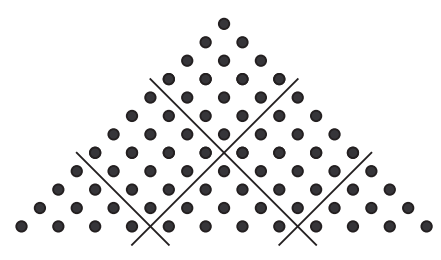

(a) $T_{12}$

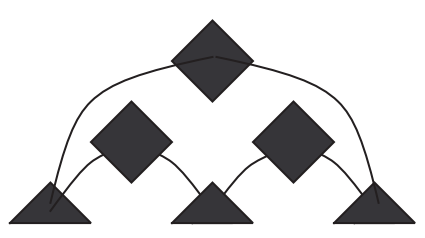

(b) $T_{3}$ with $T_{4}$ and $D_{4}$ as supernodes

Fig. 6. Creating a scheme for $T_{12}$ using schemes for $T_{3}$ and $T_{4}$

\subsection{Minimizing the Number of Edges in the 2-Hop Scheme}

The obvious question to consider is how we choose $a$ and $b$ so that the total number of edges $e$ is minimized. The number of edges $e$ is given by the formula

$$
\begin{aligned}
e & =\frac{1}{6} a b(a(b-1)(b+4)+(a-1)(a+4)) \\
& =\frac{1}{6} a b\left(a b^{2}+3 a b-a+a^{2}-4\right)
\end{aligned}
$$

Writing $b=n / a$, we obtain $e$ as a function of $n$ and $a$ :

$$
e=\frac{1}{6} n\left(\frac{n^{2}}{a}+3 n-a+a^{2}-4\right)
$$

Differentiating $e$ with respect to $a$ we obtain

$$
\frac{1}{6} n\left(-\frac{n^{2}}{a^{2}}-1+2 a\right)=\frac{1}{6} n\left(-b^{2}-1+2 a\right)
$$

This expression evaluates to 0 when $2 a=b^{2}+1$. Hence, the minimum number of edges occurs when $2 a=b^{2}+1$. Table 3 shows how the number of edges varies for $n=12$. (The values of $2 a$ and $b^{2}+1$ are tabulated for convenience.) We see that the smallest number of edges occurs when $a=4$. In this case, $b=3$ and $2 a \approx b^{2}+1$. Notice that $a=1$ and $a=12$ are boundary cases in which the whole scheme requires one hop (since any scheme for $T_{1}$ requires 0 hops). Hence the combined scheme is equivalent to a simple 1-hop scheme.

Let us now assume that $n=a b$ and $a=\frac{1}{2}\left(b^{2}+1\right)$. (In other words, we have chosen $n$ so that the minimum number of edges will be attained.) Substituting 


\begin{tabular}{r|r|r|r|r}
$a$ & $b$ & Edges & $2 a$ & $b^{2}+1$ \\
\hline 1 & 12 & 352 & 2 & 145 \\
2 & 6 & 212 & 4 & 37 \\
3 & 4 & 172 & 6 & 17 \\
4 & 3 & 160 & 8 & 10 \\
6 & 2 & 172 & 12 & 5 \\
12 & 1 & 352 & 24 & 2
\end{tabular}

Table 3. How the number of edges varies for different 2 -hop schemes for $T_{12}$

$a=\frac{1}{2}\left(b^{2}+1\right)$ into $e$, we obtain

$$
\begin{aligned}
e & =\frac{1}{24} n\left(3 b^{4}+6 b^{3}+2 b^{2}+6 b-17\right) \\
& =\frac{1}{24} n\left(3 b^{2}\left(b^{2}+1\right)+6 b\left(b^{2}+1\right)-b^{2}-17\right) \\
& \leqslant \frac{1}{24} n(6 b n+12 n) \quad \text { since } n=a b=\frac{1}{2} b\left(b^{2}+1\right) \\
& =\frac{1}{4} n^{2}(b+2)
\end{aligned}
$$

Therefore, when $n=\frac{1}{2} b\left(b^{2}+1\right)$, there exists a 2-hop scheme for $T_{n}$ with no more than $\frac{1}{4} n^{2}(b+2)$ edges. When $b=3$, for example, we have $a=5$ and $n=15$. In other words, the best 2 -hop scheme for $T_{15}$ arises when we use copies of $T_{5}$ and $D_{5}$ as supernodes and use a 1-hop scheme for $T_{3}$ to move between the supernodes; for this construction, we have $e=265$. The upper bound derived above is $\frac{1}{4} 15.15 .5=\frac{1125}{4}$ which clearly exceeds 265 .

Since $n=\frac{1}{2} b\left(b^{2}+1\right)$, we have $b<\sqrt[3]{2 n}$. Notice that a 1 -hop scheme requires $\frac{1}{6} n(n-1)(n+4)$ edges - that is, approximately $\frac{1}{6} n^{2}(n+4)$ edges - while a 2 -hop scheme requires approximately $\frac{1}{4} n^{2}(\sqrt[3]{2 n}+2)$. The 1 -hop scheme for $T_{15}$, for example, requires 665 edges, compared to 265 for the 2 -hop scheme described in the previous paragraph.

Note that $\sqrt{n}$, while perhaps being the natural choice for $a$ (and $b$ ), is not the optimal choice. To see this, note that the number of edges $e$ for such a 2-hop scheme is given by

$$
\begin{aligned}
e & =\frac{1}{6} n(\sqrt{n}(\sqrt{n}-1)(\sqrt{n}+4)+(\sqrt{n}-1)(\sqrt{n}+4)) \\
& =\frac{1}{6} n(\sqrt{n}-1)(\sqrt{n}+4)(\sqrt{n}+1) \\
& =\frac{1}{6} n(n-1)(\sqrt{n}+4)
\end{aligned}
$$

However, $\sqrt{n}+4>\sqrt[3]{2 n}+2$. Hence, this scheme requires more edges than the optimal choice. For $n=256$, for example, we find the best choice of $a$ is 32 (and 
$b=8)$ not $a=b=16$. This is what our requirement $2 a=b^{2}+1$ would suggest, since $2.32 \approx 8^{2}+1$. The 2 -hop scheme in which $a=32$ requires 162304 edges, whereas the scheme in which $a=16$ requires 217600 .

\subsection{Other Possibilities}

Of course, we may decide that we wish to bound the number of derivation steps by some number $h \neq 2$. One obvious reason why we might do this in practice is if the storage costs of the 2-hop scheme are too high. In this case, we might split $T_{n}$ into copies of $T_{a}$ and $D_{a}$ within $T_{b}$ and define schemes that require $h_{a}$ and $h_{b}$ hops, where $h=h_{a}+h_{b}$. We can use any of the techniques described elsewhere in this paper to construct schemes that provide the desired trade-off between worst-case derivation time and the amount of public information required. A more systematic exploration of these constructions is beyond the scope of this paper and will be the subject of future work.

A second possibility is that we may require a 3-hop scheme. In this case, providing $n$ has three factors, $a, b$ and $c$, say, we can split $T_{n}$ into $b c$ copies of $T_{a}$, derive a 1-hop scheme for $T_{a}$, and then develop a 2-hop scheme for $T_{b c}$ as above.

\section{Concluding Remarks}

In this paper we have developed a number of schemes for reducing the derivation time required for keys in cryptographic schemes for implementing temporal access control. This reduction has been achieved at the expense of the addition of a small amount of public information (although the reduction from $m-1$ steps to $\log _{2} m$ described in Scheme 2 requires no additional storage). The characteristics of a number of the schemes we have described are summarized in the bottom section of Table 4 . In the remainder of this section we consider related and future work.

\subsection{Related Work}

There are two strands of closely related work. Both strands include schemes in which users may have up to three keys. We focus on schemes in which users have a single key, as they are directly comparable to our schemes. ${ }^{3}$ The relevant characteristics of comparable schemes in the literature are summarized in Table 4 .

- Atallah et al. [2] propose a number of schemes that reduce the number of edges and the maximum number of hops for $I_{m}$, using techniques they had previously developed for total orders [3].

\footnotetext{
${ }^{3}$ We noted in Sect. 2 that schemes in which users have more than one key are usually considered to be undesirable. Naturally, increasing the number of keys is sure to reduce either the public storage or the key derivation time or both.
} 
- Ateniese et al. [4] and De Santis et al. [11] propose a number of schemes that take a quite different approach, using existing work due to Thorup [14] and to Dushnik and Miller [13] to reduce the diameter of $I_{m}$.

\begin{tabular}{ll|r|r}
\multicolumn{1}{l|}{ Scheme } & Storage & Derivation \\
\hline Atallah et al. & {$[2, \S 4]$} & $\mathcal{O}\left(n^{2} \log _{2} n\right)$ & 4 \\
& {$[2, \S 4]$} & $\mathcal{O}\left(n^{2}\right)$ & $\mathcal{O}\left(\log _{2}^{*} n\right)$ \\
\hline Ateniese et al. & {$[4, \S 4.2]$} & $\mathcal{O}\left(n^{3}\right)$ & 1 \\
\hline De Santis et al. $[11, \S 3.1]$ & $\mathcal{O}\left(n^{2}\right)$ & $\mathcal{O}\left(\log _{2} n \log _{2}^{*} n\right)$ \\
& {$[11, \S 3.1]$} & $\mathcal{O}\left(n^{2} \log _{2} n\right)$ & $\mathcal{O}\left(\log _{2}^{*} n\right)$ \\
& {$[11, \S 3.1]$} & $\mathcal{O}\left(n^{2} \log _{2} n \log _{2}\left(\log _{2} n\right)\right)$ & 3 \\
\hline Our work & Scheme 1 & $\frac{1}{6} n(n-1)(n+4)$ & 1 \\
& Scheme 2 & $n(n-1)$ & $\log _{2} n$ \\
& Scheme 3 $(b=2)$ & $\frac{3}{2} n(n-1)$ & $\frac{1}{2} \log _{2} n$ \\
& $\S 5.1$ & $\frac{1}{4} n^{2}(b+2)$ & 2
\end{tabular}

Table 4. A comparison of related work

The main distinguishing feature of our work is the focus on improved schemes that are directly relevant to the problem at hand, whereas prior work has simply applied existing techniques without considering the particular characteristics of the graph $I_{m}$ and its application to temporal access control. As a consequence, we are able to define tight bounds on storage and derivation costs, whereas related work only describes asymptotic behavior. For large values of $n$, such a description may be useful, but, for smaller (and arguably more relevant) values of $n$, our approach is more informative. Moreover, without knowing the "magic" constants hidden by the $\mathcal{O}$ notation, it is difficult to ascertain which scheme in the literature is the best to use for a particular value of $n$. Finally, we may still be able to apply some of the techniques used by De Santis et al. to further reduce the storage and derivation time for our schemes.

\subsection{Future Work}

There are two obvious areas for future work. The first is to investigate how to use the techniques described in Sect. 5 to construct $h$-hop schemes for a specified value of $h$. At the moment, we have only considered the case $h=2$. We also hope to investigate recursive schemes that use the factorization of $n$ to produce $h$-hop schemes, where $h$ is the number of prime factors in $n$.

The second strand of research would consider the application of the techniques to lattices of the form $L \times I_{m}$, where $L$ is a security lattice in the usual information flow sense. These types of lattices - which provide a cryptographic 
way of enforcing temporal information flow policies - have been considered by Crampton [8], as well as De Santis et al. [4,11] and by Atallah et al. [2].

\section{References}

1. S.G. Akl and P.D. Taylor. Cryptographic solution to a problem of access control in a hierarchy. ACM Transactions on Computer Systems, 1(3):239-248, 1983.

2. M.J. Atallah, M. Blanton, and K.B. Frikken. Incorporating temporal capabilities in existing key management schemes. In Proceedings of the 12th European Symposium on Research in Computer Security, pages 515-530, 2007.

3. M.J. Atallah, K.B. Frikken, and M. Blanton. Dynamic and efficient key management for access hierarchies. In Proceedings of 12th ACM Conference on Computer and Communications Security, pages 190-202, 2005.

4. G. Ateniese, A. De Santis, A.L. Ferrara, and B. Masucci. Provably-secure timebound hierarchical key assignment schemes. In Proceedings of 13th ACM Conference on Computer and Communications Security, pages 288-297, 2006.

5. D.E. Bell and L. LaPadula. Secure computer systems: Unified exposition and Multics interpretation. Technical Report MTR-2997, Mitre Corporation, Bedford, Massachusetts, 1976.

6. E. Bertino, P.A. Bonatti, and E. Ferrari. TRBAC: A temporal role-based access control model. ACM Transactions on Information and System Security, 4(3):191223, 2001.

7. E. Bertino, B. Carminati, and E. Ferrari. A temporal key management scheme for secure broadcasting of XML documents. In Proceedings of the 8th ACM Conference on Computer and Communications Security, pages 31-40, 2002.

8. J. Crampton. Cryptographically-enforced hierarchical access control with multiple keys. Journal of Logic and Algebraic Programming, 2009. To appear: electronic preprint available from doi:10.1016/j.jlap.2009.04.001.

9. J. Crampton, K. Martin, and P. Wild. On key assignment for hierarchical access control. In Proceedings of 19th Computer Security Foundations Workshop, pages 98-111, 2006.

10. B.A. Davey and H.A. Priestley. Introduction to Lattices and Order. Cambridge University Press, Cambridge, United Kingdom, 1990.

11. A. De Santis, A.L. Ferrara, and B. Masucci. New constructions for provably-secure time-bound hierarchical key assignment schemes. In Proceedings of 12th ACM Symposium on Access Control Models and Technologies, pages 133-138, 2007.

12. D.E. Denning. A lattice model of secure information flow. Communications of the ACM, 19(5):236-243, 1976.

13. B. Dushnik and E.W. Miller. Partially ordered sets. American Journal of Mathematics, 63(3):600-610, 1941.

14. M. Thorup. Shortcutting planar digraphs. Technical Report 93-60, DIMACS, 1993. 